\title{
A Cost Effective Route Guidance Method for Urban Areas Using Histograms
}

\author{
Hesham El-Sayed, ${ }^{1}$ Gokulnath Thandavarayan, ${ }^{1}$ and Yasser Hawas ${ }^{2}$ \\ ${ }^{1}$ College of Information Technology, United Arab Emirates University, Al Ain, UAE \\ ${ }^{2}$ College of Engineering, United Arab Emirates University, Al Ain, UAE \\ Correspondence should be addressed to Hesham El-Sayed; hesham.elsayed@gmail.com
}

Received 30 June 2016; Accepted 30 October 2016; Published 10 January 2017

Academic Editor: Pietro Manzoni

Copyright (c) 2017 Hesham El-Sayed et al. This is an open access article distributed under the Creative Commons Attribution License, which permits unrestricted use, distribution, and reproduction in any medium, provided the original work is properly cited.

\begin{abstract}
Analysing shortest path for real time traffic environment is crucial with dynamic updating. VANET technology can be used for analysing traffic but generates a huge amount of data to be exchanged, which demands more processing power and resources. In this paper, a new histogram-based route guidance algorithm (HBA) has been proposed based on light weight processing. The proposed algorithm enables selecting the shortest path between any source and destination using the histogram models, which capture the higher order distribution function of the number vehicles in every lane. Furthermore, the histogram model is used to estimate the traffic delays at intersections and roundabouts. The data entity collection through sensors used for histogram modelling is presented in detail. The experimental results show that the proposed algorithm provides a good prediction of road traffic status and a better solution for the congestion problem in the urban areas.
\end{abstract}

\section{Introduction}

Due to the growth of urban areas [1-3], the vehicle density per area square mile has been increased tremendously and thus impacts the roadway trajectory directly to have more congestion and heavy traffic jams. This challenge cannot be solved only by expanding the existing road traffic infrastructure by itself and it may be infeasible with restricted roads. To remedy congestion and traffic jams, it is important to take precaution measures and the existing roadways should be utilized properly. At the moment, Intelligent Transportation System [4-7] (ITS) is becoming an increasingly important element in the road traffic system. Intelligent transport system includes wireless automated technologies to integrate with the vehicles, drivers, and other infrastructures to communicate with each other to have better driving experience. This system helps to optimize transportation travel time [8$10]$ and enables finding desired routes for the vehicles. For example, when implementing ITS in roadways it alerts the drivers from a potential traffic jam and provides the best shortest path [11-13] to the destination. When comparing with the static approach the best shortest path likely to be evaluated from the dynamic approach which analyses the real time traffic situations [14]. Informing traffic patterns $[15,16]$ in prior makes the trip more robust. Precautious measure congestion based cost function should be estimated for every lane to predict the best possible route from source to destination $[17,18]$.

In general, the cost function can be derived from the past road traffic traces. Due to the large participant, the data generated from vehicles and infrastructure will be substantially large, thus making the system unstable. However, it requires heavy investment in terms of computation and power factors.

In this paper, we consider the abovementioned problem and propose a road traffic model which analyses the vehicles travelling in roads and queuing up in different road cross junctions. The proposed model captures the arrival rate distribution in VANET and build histograms on a time window basis to derive the shortest path for every source to destination trip. The paper is organised as follows: Section 2 discuss the existing works of route guidance in ITS environment. Section 3 describes the proposed algorithm and the 
queuing modelling. Section 4 presents the network modelling and the route guidance strategy is discussed in Section 5. In Section 6, the performance analysis is discussed with various scenarios. Finally, Section 7 concludes the paper.

\section{Related Work}

Congestion in roadways has been identified as a major cause for the problems created in urban areas, whereby creating distress to travellers. Many researchers have been prudent to analyse the congestion problem in urban areas. Real time traffic data gives the insight of the real time events and can be used in classifying congestion [19] for route forecasting. Such technology would also lead to improvements in existing transportation while reducing the cost of new infrastructure. Barrachina et al. [20] presented a paper for continuously estimating the vehicular density using V2I for traffic redirection. It allows the intelligent transport system to estimate the density by computing the number of beacons received per roadside unit. Pan et al. [21] proposed proactive five different rerouting algorithms that include the dynamic shortest path (DSP), the A* shortest path with repulsion $(\mathrm{AR} *)$, the random $\mathrm{k}$ shortest path $(\mathrm{RkSP})$, the entropybalanced kSP (EBkSP), and the flow-balanced kSP (FBkSP) and studied the effective travel time. However, the algorithms were active all the time and thus the vehicles trend to take some of the shortest route more often and leaving behind other routes. This could again make the network unstable due to congestion in some particular roads. Rajabi-Bahaabadi et al. [22] propose an efficient path finding algorithm that is suitable for road networks using nondominant sorting algorithm and its parameters are enhanced by the Taguchi method. Nevertheless, one limitation to the V2I architecture is that it contains a single point-of-processing, meaning that the central server process a large amount of data in real time that must be difficult by the server when all of the vehicles are sending speed and location data, as well as querying for fastest paths (or other information). The proposed traffic modelling architecture attempts to reduce these limitations by offering the benefits of the histogram graphs to the vehicles. In the proposed algorithm, the transportation network is broken into individual lanes, each of which consists of one or more edges. The lanes are preconfigured with sensors to observe the pattern and transfer the data to the intersections. The vehicles are counted by the sensors on a window basis and transmitted wirelessly to the corresponding intersection, which groups all the data for building histograms, so the centralized server is avoided and a decentralized methodology is implemented for processing the histograms to find the shortest path. Desai et al. [23] took a congestion problem in roadways and proposes a multiagent based approach using intervehicular communications (IVC). In order to estimate the best possible route for a trip, key traffic information should be obtained from many vehicles as travelling along the way; this could be a problem when there is no such vehicle travelling for a specific destination and the system may predict a high demanding route and thus could lead to more congestion. Pan and Borcea [24] propose a rerouting strategy using a decentralized approach method. It uses vehicular ad hoc networks to exchange information between vehicles to estimate the best path. The data overload could be substantially high due to its message exchange rated between the server and internet communication. Due to the handling of huge data from the entire network, high resource utilization is required in this methodology. The proposed traffic modelling architecture attempts to reduce these limitations by offering the benefits of the histogram graphs to the vehicles. In the proposed algorithm, the transportation network is broken into individual lanes, each of which consists of one or more edges. The lanes estimate the congestion identically by counting the vehicles and then exchange the information with other RSU units. The model uses less data exchange rate as it does not indulge vehicles in route estimation process. The proposed model is explained in detail in further chapters.

\section{Proposed Algorithm}

The proposed histogram-based algorithm (HBA) is divided into five stages: (1) Traffic Monitoring, (2) Histogram Computing, (3) Queuing Model (4), Network Modelling (V2I), and (5) Route Guidance. The hierarchy of each stage in the proposed algorithm is shown in Figure 1. The description of each stage is presented in the following sections.

3.1. Traffic Monitoring. Modelling and analysis of the road traffic in a statistical way give the insight of understanding the relationship between the higher order distribution function and the roadway navigation algorithms. Every lane has its own characteristics based on the vehicle injection behaviour over the time period, for example, some intersections will have more congestion during the office hours and some intersections will be more congested during the school timings. The congestion happening in urban areas is heterogeneous and changes over the period of time. In the proposed algorithm, this behaviour of changing patterns has been studied and taken into consideration. The traffic flow can be predicted by observing the vehicles travelling in every lane. To estimate the traffic flow of individual lanes, a density function should be derived as follows. ITS uses wireless sensor nodes that is intended for monitoring the road environment. The major advantage of using wireless sensor nodes in the road side will enable to capture the real time behaviour of roadways. The sensors will sense and collect data from a certain lane, process them, and transmit them to the RSU (Road Side Unit). Sensors are used in every lane to count the number of vehicles on a window basis. This information is used to build histograms by the RSU and used for further processing. As shown in Figure 2, a signalized lane is connected by two intersections in each end is selected and taken as an example. Each lane has its own sensors coupled with the corresponding RSU. It is done by providing unique identification code (e.g., SeqNum) for both sensors and RSU units. When the information is broadcasted, only the corresponding RSU will process the data based on the unique code. In the diagram, we clearly see that when the sensors transmit the data, it is received only by the corresponding RSU unit. 


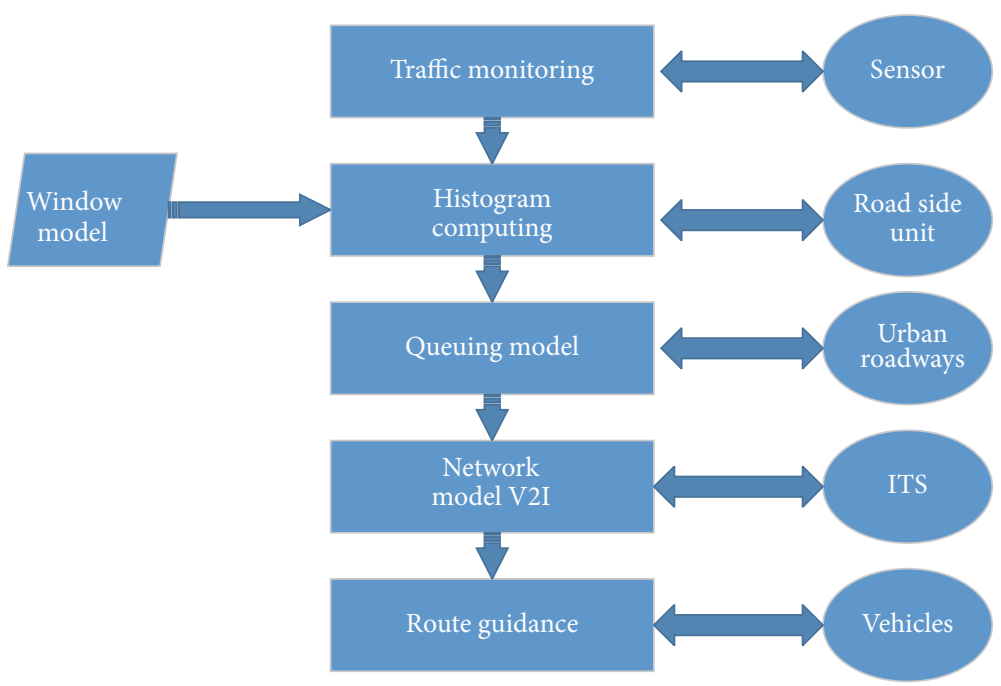

FIgURE 1: Proposed algorithm.

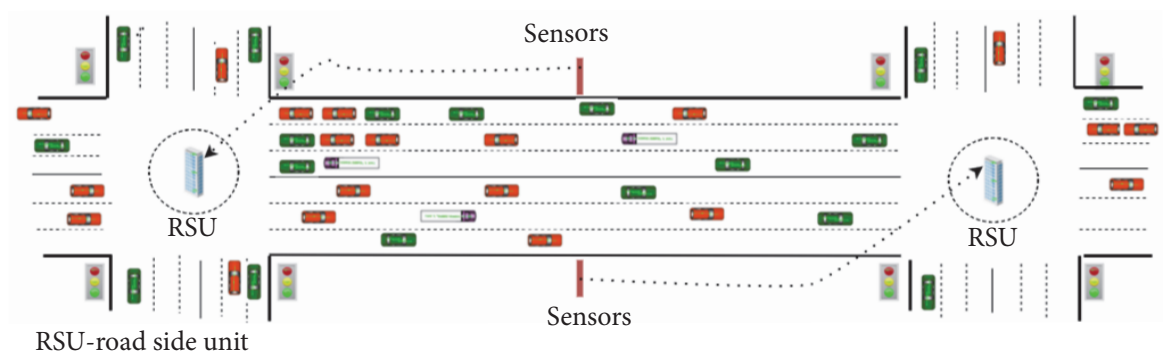

FIGURE 2: Real time estimation of vehicle density.

The principle behind the estimation of density is to find the cost function for all individual lanes. Imagine there can be different routes exist between a source and destination. To select a best shortest path, we need to estimate the cost of every route based on the congestion by using the real time data and cost can be a factor that estimates the total travelling time in that particular lane.

3.2. Histogram Computation. In this paper, we use histograms to model traffic distribution in urban areas. We sense the road traffic flows on every lane for a predefined period (also known as an observation period) and build histograms on window basis. A new histogram per lane is built in every observation period $O$, where the length of the observation period depends on the lane characteristics. During every observation period, the number of vehicles in each lane is counted over $N$ equal sampling periods of length $T$ each, where $N=O / T$. The frequency of these numbers is then computed to construct the histogram model.

As the number of vehicles in any sampling period can vary over a large set of integers $I:=\{0,1, \ldots, K\}$, where $K$ is the maximum number of vehicles that can be held in the street, we divide this $[0 \cdots K]$ range into a limited number of
TABle 1: Example of a Group Probability Distribution for vehicles during an observation period $O$.

\begin{tabular}{lcccc}
\hline $\begin{array}{l}\text { Class } \\
\text { number }\end{array}$ & $\begin{array}{c}\text { Class } \\
\text { range } \\
\left\{C_{i}^{-}, C_{i}^{+}\right\}\end{array}$ & $\begin{array}{c}\text { Class } \\
\text { midpoint } \\
C_{i}\end{array}$ & $\begin{array}{c}\text { Frequency } \\
\text { of } \\
\text { occurrence }\end{array}$ & $P\left(C_{i}\right)$ \\
\hline 0 & $\{0,10\}$ & 5 & 8 & 0.2 \\
1 & $\{10,20\}$ & 15 & 16 & 0.4 \\
2 & $\{20,30\}$ & 25 & 6 & 0.15 \\
3 & $\{30,40\}$ & 35 & 6 & 0.15 \\
4 & $\{40,50\}$ & 45 & 4 & 0.1 \\
5 & $\{50,60\}$ & 55 & 0 & 0 \\
\hline
\end{tabular}

equal width classes $N_{c}$ and compute the grouped probability distribution function over each class. Table 1, for example, shows the distribution of the number of vehicles in one lane over $N_{c}=6$ classes during an observation period $O=10$ minutes and sampling period $T=15$ seconds. The number of sampling periods $N=10 * 60 / 15=40$ sampling period. For each class $i$, the table shows the lower limit $C_{i}^{-}$, the upper limit $C_{i}^{+}$, the middle point $C_{i}$, the number of intervals during which this class range was encountered, and the probability 


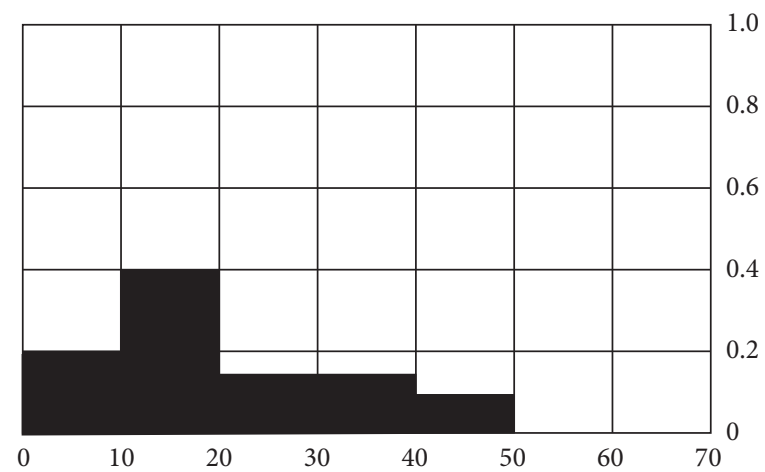

Figure 3: Histogram for traffic density during a single observation Period $O$.

of occurrence of this class $P\left(C_{i}\right)$ during the observation period. The probability $P\left(C_{i}\right)$ is calculated as shown in

$$
P\left(C_{i}\right)=P\left(\frac{\text { No of sampling periods with vechicles counts in the range }\left(C_{i}^{-} \leq C_{i} \leq C_{i}^{+}\right)}{\text {Total number of sampling periods }}\right)
$$

The histogram is then derived from the table to measure the congestion density of a particular lane during an observation window as shown in Figure 3. To ensure that the statistical model of histogram is suitable for practical use, we assume that the number of vehicles within an observation period/window is a stationary process and independent from window to window.

In general, any statistical histogram $C$ can be defined using two main attributes:

(i) A set of quantized number of cars per class $\bar{C}$, which can be derived by averaging the upper and lower limits of number of cars in each class

(ii) A set of probabilities $P$ corresponding to $\bar{C}$ :

$$
C=(\bar{C}, P)= \begin{cases}\bar{C}=C_{i}, & i=0,1, \ldots, N_{c}-1, \\ P=P\left(C_{i}\right), & i=0,1, \ldots, N_{c}-1,\end{cases}
$$

where $N_{c}$ is the number of classes.

The histogram presented in this paper represents the statistical distribution of vehicles in an observation window basis against their corresponding probabilities (or frequencies). The number of vehicles can be easily obtained using sensors along lanes, and the histograms can be computed using the Road Side Units (RSUs) and then disseminated to vehicles moving on the road through an ad hoc based infrastructure-to-vehicle network.

3.3. Queuing Models for Vehicular Road Traffic. Urban roadway commonly has three types of road junctions [25], including road junction with traffic-signals, road junction with round-about, and road junction with fly-over. Initially, we consider a road junction with traffic signal interconnected by two-way roads. Let $r(t) \mid t \in\{1,2, \ldots, N\}$ be the vehicle speed, in kilometre per hour, during a sampling period $t$. According to the 2-second rule for road safety, the minimum distance between consecutive vehicles on the street is given by $d(t)=(r(t) * 1000 * 2) / 3600$ meters. If $\bar{V}$ is the average length of vehicle in meters, then vehicles from the street will move into the road cross junction at a rate of $D(t)=r(t) /(d(t)+$ $\bar{V}) * 1000 / 3600$ vehicles per second. Furthermore, if $W$ is the length of the road between two adjacent road junctions, then the maximum number of vehicles that can be held by the road during a sampling period $t$ is given by $K(t)=W /(d(t)+\bar{V})$ vehicles.

The road traffic signal is modelled as a Markov modulated Bernoulli process (MMBP) consisting of ON state and OFF state. The $\mathrm{ON}$ state represents the green signal and the OFF state represents the red signal (in this case, yellow signal is not taken into account). If vehicles from different directions are equally accessing to the road cross junction, which is controlled by traffic lights with equal access time operating on opposite two-way traffic basis, then vehicles from one direction moves into the inter-section at an average rate of $D(t) / 2$ vehicle per second. Hence, the queue at the cross junctions can be modelled as finite buffer $\mathrm{HD} / D / 1 / K$ queue. Where, HD stands for the histogram Deterministic Interarrival distribution that represents vehicle flow in the queue, $D$ represents the deterministic process of vehicles moving from the street into the cross junction at a rate of $D(t) / 2$ and $K(t)$ represents the maximum number of vehicles that can be held by the street during a sampling period $t \in\{1,2, \ldots, N\}$. The server discipline is first come first served.

The usage of the $\mathrm{HD} / D / 1 / K$ queuing model can be justified as follows. From statistical analysis point of view, the performance of the queue depends on the interarrival of 
vehicles moving on the street, which is relatively determined by the number of vehicles moving into the system within a single unit of time. Although the number of vehicles moving into the queuing system is time variable, it is much slower comparing to the time for a vehicle across the queue. In this case, if the histogram window is chosen properly, the vehicle interarrival process within the window can be treated as a stable process. We also assume that vehicles are independent of each other and move from road into the cross junction at a constant speed since it is determined by the road traffic signal.

Likewise, a street connected with a round-about junction is modelled as finite buffer $\mathrm{HD} / D / 1 / K$ queue, where $D$ represents the deterministic process of vehicles moving from street into the round-about junction at an average rate of $D(t) / 4$. In other words, we assume that vehicles randomly access the round-about junction from 4 directions with equal distribution with a mean value of $D(t) / 4$. Similarly, a road junction with fly-over can be modelled as free-access buffer without traffic control mechanism. Therefore, a roadway in urban city can be modelled as a tandem chain of $\mathrm{HD} / D / 1 / K$ queues [26].

Let $V(t) \quad \mid t \in\{1,2, \ldots, N\}$ represent the number of vehicles that enter the street during the $t$ th sampling period. Let $\tau[t]$ be the number of vehicles that exit from the street during the same period. The vehicle input flow is supplied through a street/buffer of finite capacity. The pending vehicles that cannot move from the street and enter the road intersection during the observation period will be queued in the street. The system is assumed to be in stationary if the pending traffic convergences to a finite value. Let $Q[t]$ represents the queue length in sampling period $t \in\{1,2, \ldots, N\}$. In the classic analysis of $M / D / 1 / K$ queuing system, the $Q[t]$ can be expressed as follows:

$$
Q[t]=\emptyset_{0}^{K}(Q[t-1]+V(t)-\tau[t]),
$$

where Operator $\emptyset$ limits the queue lengths so that they cannot either underflow or overflow the lane capacity $K(t)$ in the $t$ th sampling period. The $\emptyset$ operator is defined as shown in

$$
\emptyset_{a}^{b}(x)= \begin{cases}0, & \text { for } x<a, \\ x-a, & \text { for } a \leq x<b+a, \\ b, & \text { for } x \geq b+a\end{cases}
$$

Since the number of vehicles moving from the street into the cross junction during the $t$ th sampling window is given by $D(t) \times T$ vehicles, then the number of vehicles that exit from the street during the same period is given by $\tau[t]=D(t) / 2 \times$ $T$, where $T$ is the duration of the sampling period. Likewise, if the cross junction is a round-about, then $\tau[t]=D(t) / 4 \times T$. When $V[t] \leq \tau[t]$, the buffer size is not increased. Otherwise, the buffer occupancy increases until queuing buffer is full. Therefore, equation (5) can be expressed as

$$
\begin{aligned}
Q[t] & =\emptyset_{0}^{K}(Q[t-1]+V(t)-\tau) \\
& =\emptyset_{\tau}^{K}(Q[t-1]+V(t)) .
\end{aligned}
$$

The foundation of the histogram method consists of eliminating the time dependence of $V(t)$ in the previous

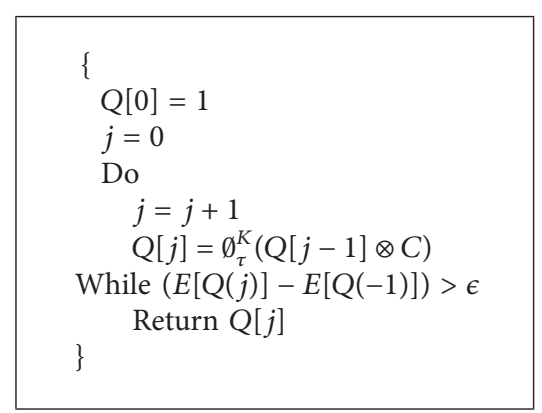

Algorithm 1: Algorithm $\operatorname{HBSP}(C, \bar{\tau}, \bar{K})$.

expression and replacing it by a discrete random variable that describes the arrival process [27]. As our traffic model assumes that traffic is stationary, that is $C=V(n) \forall n \in N$, (5) can be transformed into the statistical equation (6):

$$
Q[n]=\emptyset_{\tau}^{K}(Q[n-1] \otimes C) .
$$

The bound operator $\emptyset_{\tau}^{K}$ is the statistical generalization of the previously defined $\emptyset_{a}^{b}$ operator. $C$ is the statistical histogram defined in (2). The operator $\otimes$ stands for the standard statistical convolution, which is described as follows. If $X$ and $Y$ are two independent random variables with $n$ and $m$ intervals, respectively, the convolution $X \otimes Y$ is a new random variable $Z$ with $n+m-1$ intervals and

$$
P_{Z}[i]=\sum_{k=0}^{i} P_{X}(i-k) \times P_{Y}(k) .
$$

The steady state probability of the queue length can be calculated using Algorithm 1, where C: arrival process (histogram) as defined in (2), $\bar{\tau}$ : service rate class of $\tau, \bar{\tau}=\tau / L_{c}$, where $L_{c}$ is the class length, $\bar{K}$ : the maximum queue length class of $K, \bar{K}=\left\lfloor K / L_{c}\right\rfloor$, where $L_{c}$ is the class length, and $E(X)$ : the mean value (or expectation) of gpd $X$.

Once the steady state probabilities of the queue length are calculated, the expected queue length can be calculated as follows:

$$
E(Q)=\sum_{i=0}^{K} P\left(q_{i}\right) * i
$$

In the case of a cross junction with an output rate of $\tau$ and a queue length characterized by a gpd $Q$, the queuing delay $W$ is proportional to $Q$ and has the same pmf, where $W=1 / \tau *$ $Q$. Therefore, the expected queuing delay at each intersection can be calculated as follows:

$$
E[W]=\frac{1}{\tau} * E[Q] .
$$

\section{Network Modelling (I2V)}

The network modelling for the proposed algorithm uses the infrastructure-to-vehicle (I2V) communications for transferring the histograms across the network. Once an RSU 


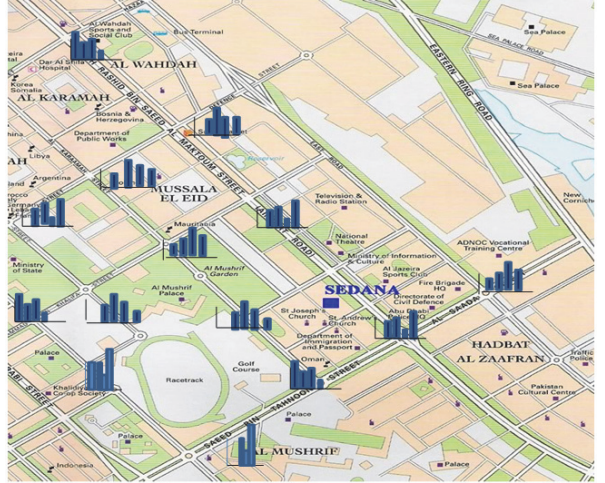

(a)

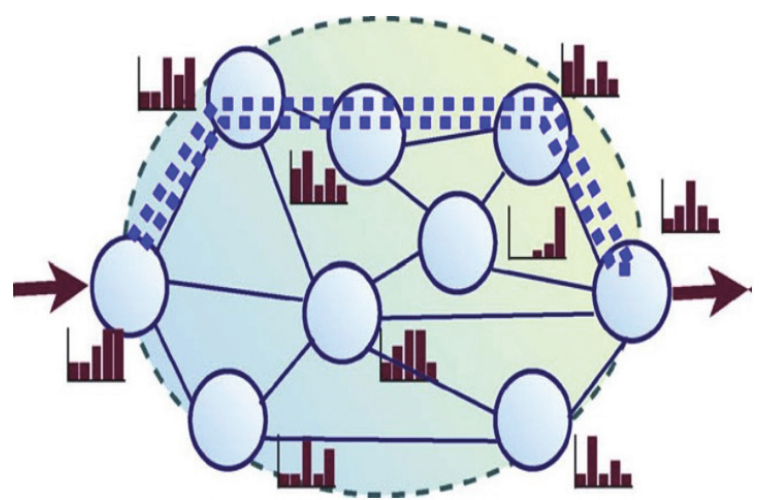

(b)

Figure 4: (a) Histogram map of Abu Dhabi. (b) Shortest route path.

computes the histogram for a corresponding lane, it informs the other RSU units to build up the histogram map of the entire network. Finally, this information is given to the vehicles travelling in the network and a sequence is added along with it. The vehicles cache the information and verify the sequence number; if the information is updated then the new information is processed and the map is updated with new histogram. Figure 4(a) shows an experimental result of the statistical histograms built in the city of Abu Dhabi obtained by on-side road traffic sensor system.

\section{Route Guidance}

As discussed in the above scheme, the histograms were computed in every lane. From this information, we can find the shortest path from source to destination across the network as shown in Figure 4(b). The proposed model adapts Dijkstra's algorithm to find the shortest path. In the proposed model, the road networks are considered as a weighted graph model, the lanes are perceived as edges, and the intersections are marked as nodes. The weight of each link, in other words the link cost, is computed as follows: LinkCost $=$ Linklenght/Speed + Queuing delay, where the Queuing delay is derived from (8). The histograms are computed across every lane and the link density function is updated periodically. The vehicles are routed according to the lane weights.

\section{Performance Metrics}

The performance analysis of the HBA-based algorithm is compared with two different existing algorithms namely, the intervehicular communication (IVC) [28] algorithm and the optimal dynamic shortest path algorithm (SPA). The working model of IVC algorithm introduces the concept of searcher vehicle and candidate vehicle, whose origin node matches the destination node of the searcher vehicle which travels in the opposite direction. SPA algorithm computes the shortest path by exchanging traffic information between these vehicles using V2V communication.
In the SPA-Dynamic algorithm, the cost of a link is computed by monitoring the traces of the vehicles across the entire network. Hence, the link cost gets updated with every single vehicle passing through the link. All links' costs are disseminated in real time to all vehicles in the network so that they can compute the shortest path to destinations. The SPA-Dynamic approach is infeasible in practice as it demands dissemination of continuous real time data in VANET environment. Moreover, this algorithm also consumes more time and requires high resource utilization. Therefore, the SPADynamic algorithm is considered to be an optimal algorithm in simulation (but not in practice). However, we are interested in adding the SPA-Dynamic approach in our performance analysis to prove the efficiency of the proposed algorithm and to depict how close the proposed algorithm can perform with the theoretical model that holds the best optimal results.

A microscopic simulator I-Sim [29] is employed to perform the comprehensive comparative analysis for the proposed system. This simulator effectively creates urban roadway traffic scenarios and generates vehicle trips randomly. The microscopic visualization of the simulator enables in analysing the traffic behaviour of roadways on a broader percept. While running the simulation, every vehicle stores all individual road trip information from source to destination, which enables determining the efficiency of the proposed algorithm. This simulator is inbuilt with a car following technique that adapts a finite state model which determines the acceleration/deceleration rate, velocity, and the distance between the vehicles. The simulator is efficient enough to designing the wide range of roadways that reflect the urban roadway system.

To study the effectiveness of the proposed system, a grid network shown in Figure 5 is used to model the urban roadway. In the topology, the grid has 49 nodes and each node acts as an intersection. The edge between any two nodes acts as a lane and its length varies according to the scenario. In each scenario, the simulator generates the vehicles randomly and the vehicle burst rate is set pertaining to the given scenario. While running the simulation, the vehicle routes are computed based on the link cost and the routes are updated in the OD Matrix. If the congestion increases in the route lane, 


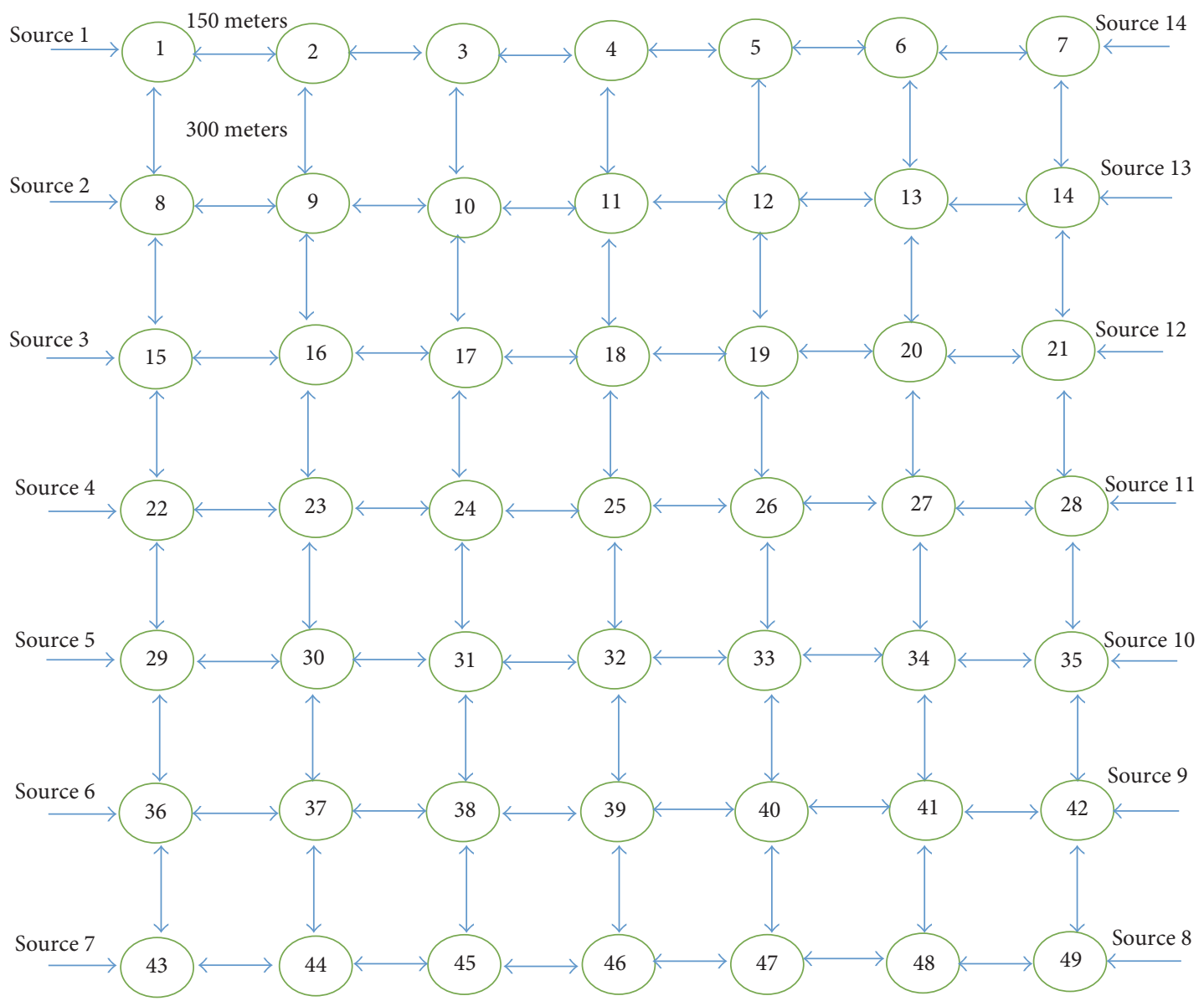

FIgURE 5: The network topology used during simulation.

a new route is computed based on the histogram model and the vehicles are rerouted with the updated shortest path.

As shown in Table 2, different scenarios where created to study the effectiveness of the proposed algorithm by varying the source volume injection rate, lane length, and maximum speed limit.

The total number of origins in the grid network is set as 14 sources, in which the vehicles start their trip randomly. In order to simulate the real time urban traffic, only 28 intersections are marked as destinations. The vehicles start the trip from any one of the sources and reach the assigned destination by taking the shortest route. Every link connected between intersections will have 3 lanes and same speed limit. Every scenario is simulated for 60 minutes and the average travel time is computed.

6.1. Source Volume Scenario. In this scenario, the link length and the lane speed are kept as constant and the volume demand differs with different vehicle burst rate from the source. Three scenarios were carried out using the link speed of $80 \mathrm{~km} / \mathrm{hr}$; horizontal and vertical lane lengths are $300 * 150$. Figure 6 shows average travel time versus the source volume generation.

In the experiment, it shows that the average travel time taken by every algorithm increases as the source volume increases. This is due to the congestion happening in the entire traffic network area. The HBA proposed algorithm gives better results than the IVC algorithm and gives close results with the SPA, which is considered to be an optimal algorithm. However, the SPA approach is infeasible in practice as dissimilating real time data continuously and instantaneously in VANET environment is impossible due to time and computation constrains.

6.2. Link Speed Scenario. In general, as the link speed increases, the network accommodates more vehicles. Furthermore, the relative network productivity increases with the increase of speed. Therefore, as the link speed increases, the network accommodates more vehicles. As shown in Figure 7, the performance of HBA gives good results when the lane speed increases, the vehicles traveling through the shortest path link are purely driven by histograms that are available in every lane as a cost function. Finally, an increase in speed significantly increases the efficiency of the proposed algorithm.

6.3. Link Length Scenario. Figure 8 shows the average travel time performance measure with various network sizes. For all the three scenarios, the source volume burst rate is fixed 
TABLE 2: Experimental scenario details.

\begin{tabular}{|c|c|c|c|c|}
\hline & Source volume scenario (veh/hr) & $\begin{array}{l}\text { Link speed scenario } \\
(\mathrm{km} / \mathrm{hr})\end{array}$ & \multicolumn{2}{|c|}{$\begin{array}{l}\text { Link length scenario } \\
\text { (meters) }\end{array}$} \\
\hline & $\begin{array}{c}\text { Link speed }=80 \mathrm{~km} / \mathrm{hr} \\
\text { Horizontal link length }=300 \mathrm{~m} \\
\text { Vertical link length }=150 \mathrm{~m}\end{array}$ & $\begin{array}{l}\text { Source volume }=2000 \mathrm{veh} / \mathrm{hr} \\
\text { Horizontal link length }=300 \mathrm{~m} \\
\text { Vertical link length }=150 \mathrm{~m}\end{array}$ & \multicolumn{2}{|c|}{$\begin{array}{l}\text { Source volume }=2000 \mathrm{veh} / \mathrm{hr} \\
\text { Link speed }=80 \mathrm{~km} / \mathrm{hr}\end{array}$} \\
\hline & \multirow{2}{*}{ Vehicle burst rate } & \multirow{2}{*}{ Link maximum speed } & \multicolumn{2}{|c|}{ Link distance } \\
\hline & & & Horizontal & Vertical \\
\hline Experiment 1 & 500 & 60 & 300 & 150 \\
\hline Experiment 2 & 1000 & 80 & 300 & 300 \\
\hline Experiment 3 & 2000 & 100 & 500 & 500 \\
\hline
\end{tabular}

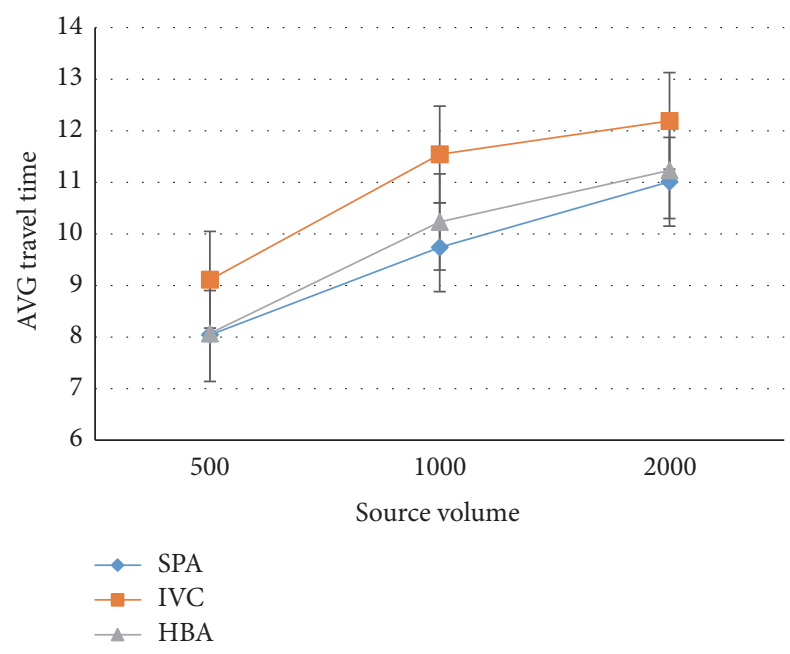

FIgURE 6: Source volume scenario.

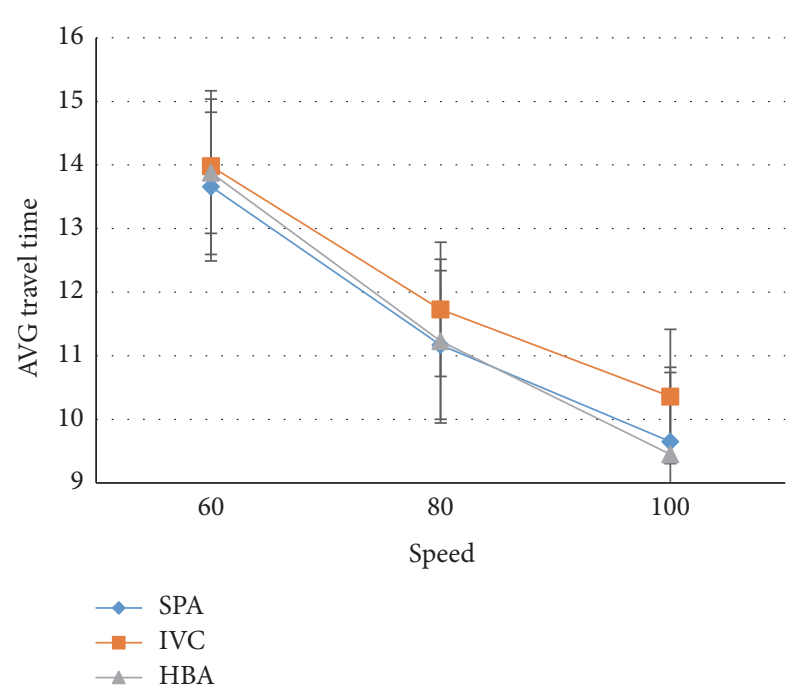

FIGURE 7: Link speed scenario.

as $2000 \mathrm{veh} / \mathrm{hr}$ and the link speed as $80 \mathrm{~km} / \mathrm{hr}$. The average travel time for HBA ranges wihtin 11.23-16.02 minutes as the travel time is directly proportional to the lane length when

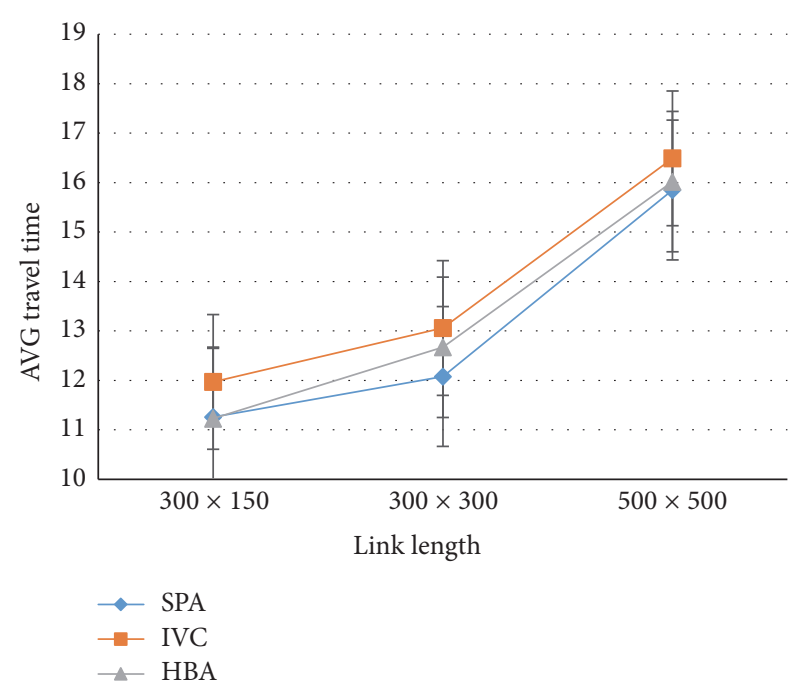

FIGURE 8: Link length scenario.

the speed is fixed. The HBA travels from source to destination with less travel time comparatively with the IVC algorithm.

By analysing the figure, it shows that when link length increases and the speed is kept as constant the relative average travel time increases. It shows that the minimum average travel time is associated with less grid length in $(300 * 150)$ scenario. This is mainly because the path is progressively determined with the ratio of the histogram graph.

\section{Conclusion}

This paper presented a novel route guidance algorithm, which forecasts traffic delays using lightweight histogram models. The histogram model extended the deterministic model, which usually describe the traffic with a discrete number of classes with a quantifying probability. Adapting the queuing model in the system makes the model more invigorate and helps to estimate the real time behaviours of roadways. A microscopic simulation is used to assess the effectiveness of the algorithm comparatively against two algorithms from literature. The performance was analysed with various scenarios reflecting the network source volumes, link speeds, and lengths. The proposed algorithm performed 
comparably with the optimal dynamic shortest path algorithm and outperformed the IVC algorithm, which results in lesser travel time from source to destination.

The results also showed that the proposed road traffic model can be easily obtained and transmitted in real time by individual vehicles while they are travelling on streets or queuing in road cross junctions and provides a good prediction on road traffic status.

The proposed model can be enhanced further by combining different histogram statistics with respect to speed, utilization, and throughput. This will be investigated in further research.

\section{Competing Interests}

The authors declare that they have no competing interests.

\section{Acknowledgments}

This research was supported by the Roadway, Transportation, and Traffic Safety Research Centre (RTTSRC) of the United Arab Emirates University (Grant no. 31R058).

\section{References}

[1] A. Storeygard, "Farther on down the road: transport costs, trade and urban growth in sub-Saharan Africa," The Review of Economic Studies, vol. 83, no. 3, pp. 1263-1295, 2016.

[2] M. Aljoufie, M. Zuidgeest, M. Brussel, and M. van Maarseveen, "Spatial-temporal analysis of urban growth and transportation in Jeddah City, Saudi Arabia," Cities, vol. 31, pp. 57-68, 2013.

[3] M. Q. Du, X. W. Jiang, and L. Cheng, "Estimating the capacity of urban transportation networks with an improved sensitivity based method," Discrete Dynamics in Nature and Society, vol. 2015, Article ID 827094, 13 pages, 2015.

[4] S. L. Toral, M. R. M. Torres, F. J. Barrero, and M. R. Arahal, "Current paradigms in intelligent transportation systems," IET Intelligent Transport Systems, vol. 4, no. 3, pp. 201-211, 2010.

[5] J. A. Sanguesa, F. Naranjo, V. Torres-Sanz, M. Fogue, P. Garrido, and F. J. Martinez, "On the study of vehicle density in intelligent transportation systems," Mobile Information Systems, vol. 2016, Article ID 8320756, 13 pages, 2016.

[6] K. N. Qureshi and A. H. Abdullah, "A survey on intelligent transportation systems," Middle East Journal of Scientific Research, vol. 15, no. 5, pp. 629-642, 2013.

[7] R. Meneguete, A. Boukerche, G. Maia, A. A. F. Loureiro, and L. A. Villas, "A self-adaptive data dissemination solution for intelligent transportation systems," in Proceedings of the 11th ACM Symposium on Performance Evaluation of Wireless Ad Hoc, Sensor, and Ubiquitous Networks, pp. 69-76, ACM, September 2014.

[8] C. Bai, Z.-R. Peng, Q.-C. Lu, and J. Sun, "Dynamic bus travel time prediction models on road with multiple bus routes," Computational Intelligence and Neuroscience, vol. 2015, Article ID 432389, 9 pages, 2015.

[9] E. I. Vlahogianni, "Optimization of traffic forecasting: intelligent surrogate modeling," Transportation Research Part C: Emerging Technologies, vol. 55, pp. 14-23, 2015.
[10] D. Tian, Y. Yuan, H. Qi et al., "A dynamic travel time estimation model based on connected vehicles," Mathematical Problems in Engineering, vol. 2015, Article ID 903962, 11 pages, 2015.

[11] R. Bauza and J. Gozálvez, "Traffic congestion detection in large-scale scenarios using vehicle-to-vehicle communications," Journal of Network and Computer Applications, vol. 36, no. 5, pp. 1295-1307, 2013.

[12] J. Ma, F. Zhou, and C. Lee, "Providing personalized system optimum traveler information in a congested traffic network with mixed users," Journal of Intelligent Transportation Systems, vol. 20, no. 6, pp. 500-515, 2016.

[13] A. Faro and D. Giordano, "Algorithms to find shortest and alternative paths in free flow and congested traffic regimes," Transportation Research Part C: Emerging Technologies, vol. 73, pp. 1-29, 2016.

[14] J.-A. Orozco and J. Barceló, "Reactive and proactive routing strategies with real-time traffic information," Procedia-Social and Behavioral Sciences, vol. 39, pp. 633-648, 2012.

[15] E. I. Vlahogianni, M. G. Karlaftis, and J. C. Golias, "Shortterm traffic forecasting: where we are and where we're going," Transportation Research Part C: Emerging Technologies, vol. 43, pp. 3-19, 2014.

[16] D. Helbing, M. Treiber, A. Kesting, and M. Schönhof, "Theoretical vs. empirical classification and prediction of congested traffic states," European Physical Journal B, vol. 69, no. 4, pp. 583-598, 2009.

[17] H. Salehinejad and S. Talebi, "Dynamic fuzzy logic-ant colony system-based route selection system," Applied Computational Intelligence and Soft Computing, vol. 2010, Article ID 428270, 13 pages, 2010.

[18] H. El-Sayed, L. Zhang, G. Thandavarayan, and Y. Hawas, "HBAhistogram based algorithm for real time route forecasting in urban area," in Proceedings of the IEEE International Conference on Communications (ICC '16), pp. 1-6, Kuala Lumpur, Malaysia, 2016.

[19] B. Morris, A. Paz-Cruz, A. Mirakhorli, and H. de la FuenteMella, "Traffic congestion classification using data mining techniques," in Economics, Social Sciences and Information Management: Proceedings of the 2015 International Congress on Economics, Social Sciences and Information Management (ICESSIM 2015), 28-29 March 2015, Bali, Indonesia, chapter 15, pp. 99-106, CRC Press, 2015.

[20] J. Barrachina, P. Garrido, M. Fogue et al., "A V2I-based real-time traffic density estimation system in urban scenarios," Wireless Personal Communications, vol. 83, no. 1, pp. 259-280, 2015.

[21] J. Pan, I. S. Popa, K. Zeitouni, and C. Borcea, "Proactive vehicular traffic rerouting for lower travel time," IEEE Transactions on Vehicular Technology, vol. 62, no. 8, pp. 3551-3568, 2013.

[22] M. Rajabi-Bahaabadi, A. Shariat-Mohaymany, M. Babaei, and C. W. Ahn, "Multi-objective path finding in stochastic timedependent road networks using non-dominated sorting genetic algorithm," Expert Systems with Applications, vol. 42, no. 12, pp. 5056-5064, 2015.

[23] P. Desai, S. W. Loke, A. Desai, and J. Singh, "CARAVAN: congestion avoidance and route allocation using virtual agent negotiation," IEEE Transactions on Intelligent Transportation Systems, vol. 14, no. 3, pp. 1197-1207, 2013.

[24] S. Pan and Borcea, "DIVERT: a distributed vehicular traffic rerouting system for congestion avoidance," IEEE Transactions on Mobile Computing, pp. 19-27, 2016.

[25] J. Shen and X. Jin, "Detailed traffic animation for urban road networks," Graphical Models, vol. 74, no. 5, pp. 265-282, 2012. 
[26] H. El-Sayed, L. Zhang, Y. Hawas, and H. El Kassabi, "A histogram-based model for road traffic characterization in VANET,' in Internet of Vehicles-Technologies and Services, vol. 8662 of Lecture Notes in Computer Science, pp. 42-45, Springer, 2014.

[27] E. Hernández-Orallo and J. Vila-Carbó, "Network queue and loss analysis using histogram-based traffic models," Computer Communications, vol. 33, no. 2, pp. 190-201, 2010.

[28] Y. E. Hawas and H. El-Sayed, "Autonomous real time route guidance in inter-vehicular communication urban networks," Vehicular Communications, vol. 2, no. 1, pp. 36-46, 2015.

[29] Y. E. Hawas, "A microscopic simulation model for incident modeling in urban networks," Transportation Planning and Technology, vol. 30, no. 2-3, pp. 289-309, 2007. 


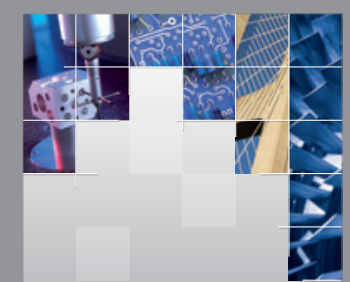

\section{Enfincering}
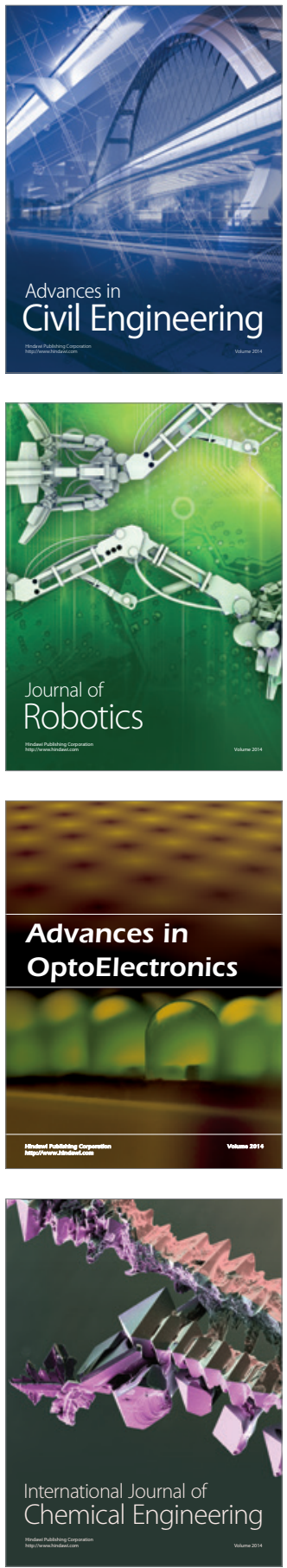

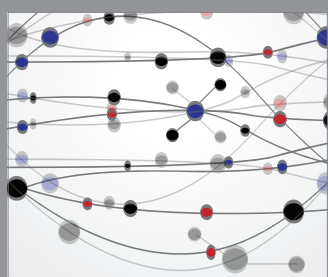

The Scientific World Journal

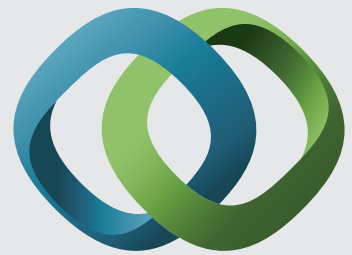

\section{Hindawi}

Submit your manuscripts at

https://www.hindawi.com
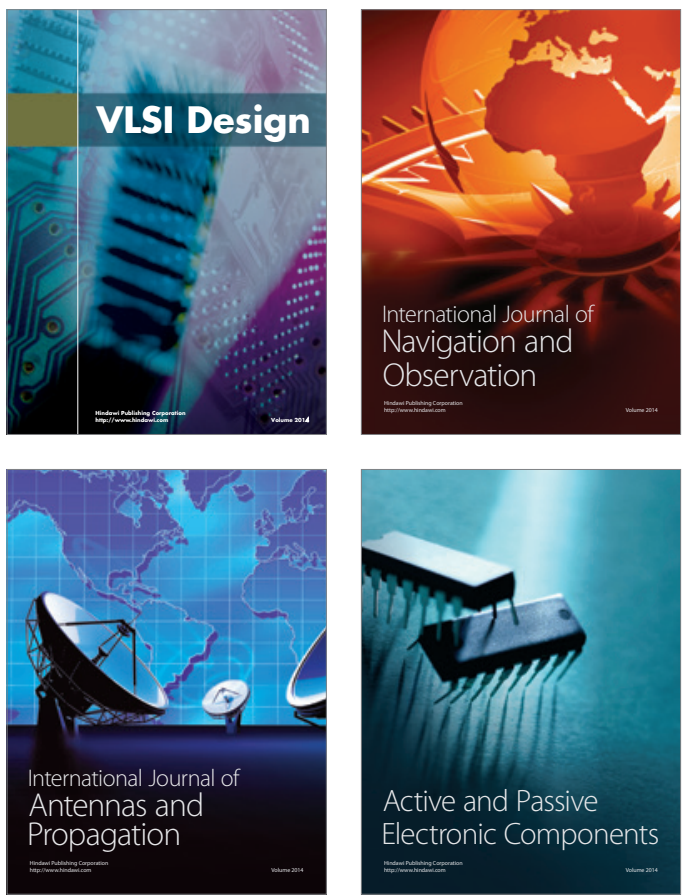
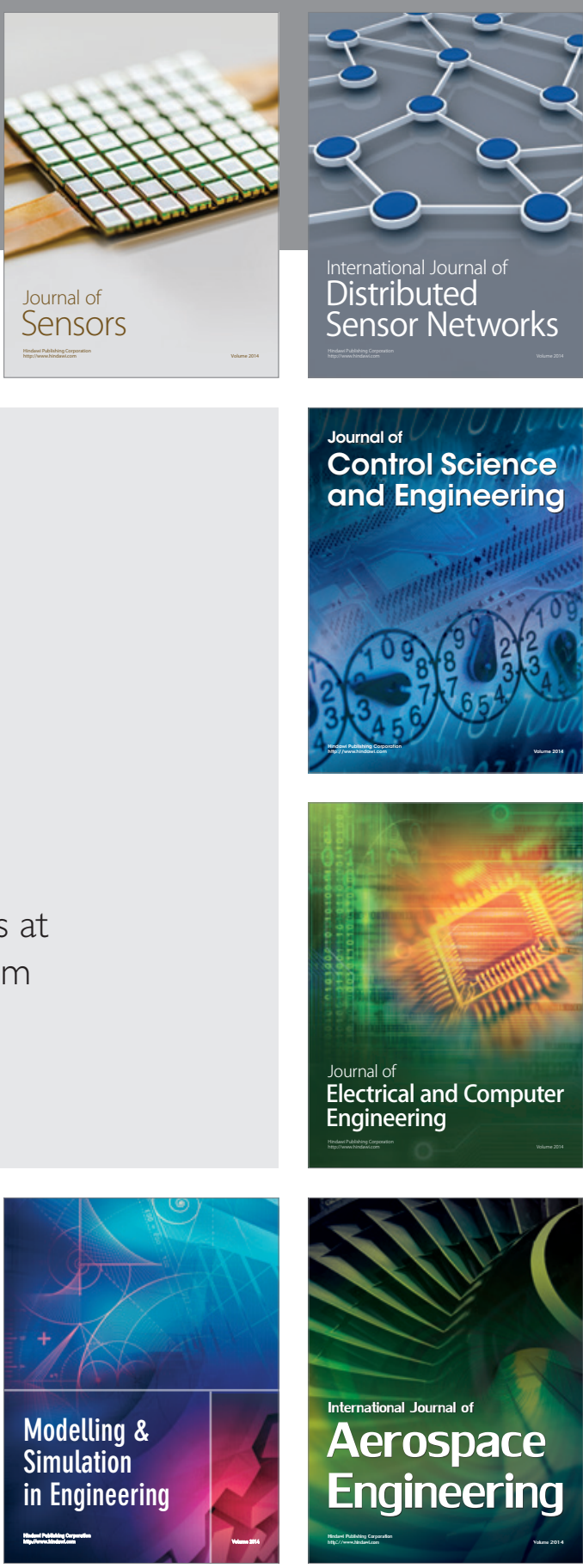

International Journal of

Distributed

Sensor Networks

$-$

Joumal of

Control Science

and Engineering
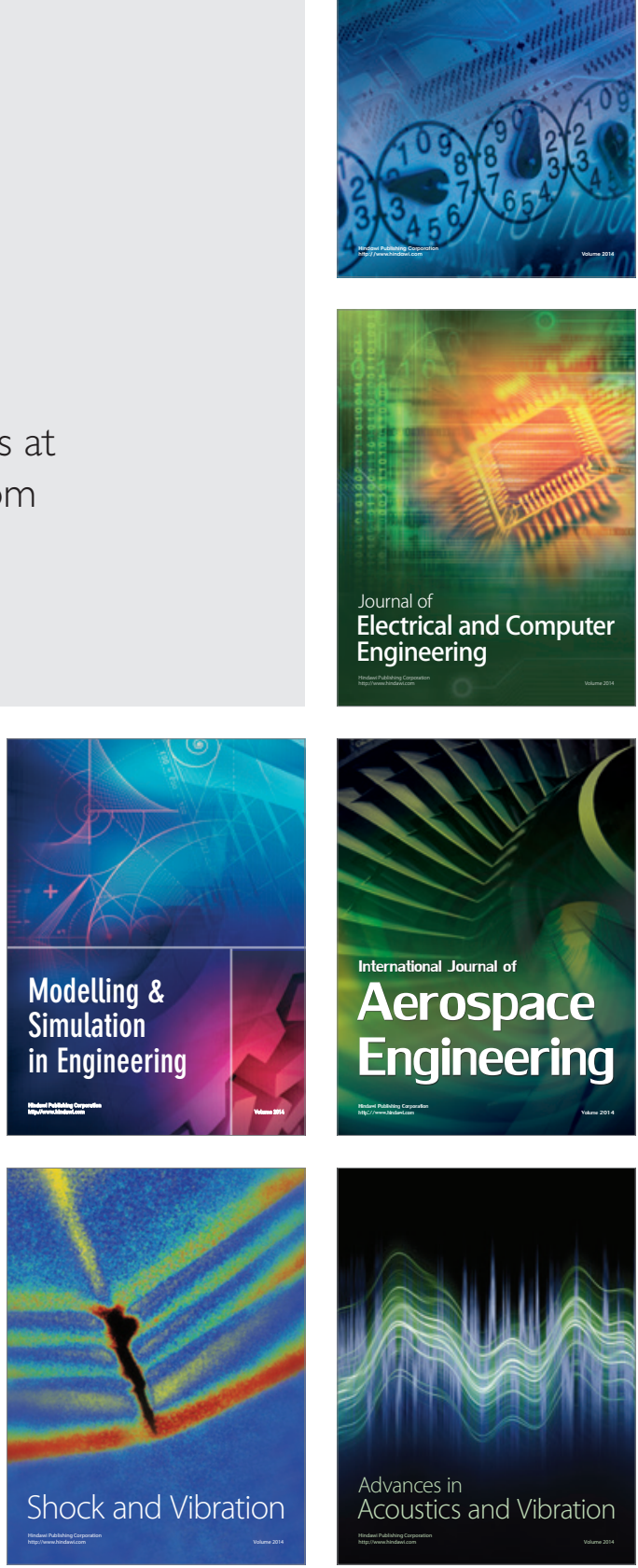